\title{
Disseminated Penicillium marneffei infection recurrence in a non-acquired immune deficiency syndrome patient: A case report
}

\author{
ZHIXUE ZHANG, FANGXU TAO, YINING LI, YUDONG XIAO, ZHISHU ZHANG and JUN LIU \\ Department of Radiology, The Second Xiangya Hospital of Central South University, Changsha, Hunan 410000, P.R. China
}

Received June 1, 2016; Accepted July 7, 2016

DOI: $10.3892 / \mathrm{mco} .2016 .1038$

\begin{abstract}
Penicillium marneffei is a rare deep tissue fungal infection causing an endemic in Southeast Asia. This infection causes penicilliosis disease and is more common in patients who are immunocompromised. To date, no cases of $P$. marneffei infection relapse following treatment have been reported. A 36-year-old patient attended our hospital as a result of intermittent fever, cough, shortness of breath and multiple soft lesions located on the face, arms, neck and trunk. The medical radiological examination of the lung revealed multiple patchy exudative shadows, thick-walled hollow inner part of the visible lesions, bilateral pleural and pericardial effusion. Assessing the skull and right collarbone, bilateral thoracic ribs and a plurality revealed numeorus lesions with reduced bone destruction and revealed that the patient was positive for P. marneffei infection. After 6 months of antifungal therapy, pulmonary symptoms and the surface lesions of the patient rapidly disappearance and the physical condition markedly improved. The patient did not attend a follow-up and stopped antifungal treatment. In February 2016, the patient presented with left breast and subcutaneous soft tissue mass of the head and neck. Fungal culture results revealed that the patient was again positive for P. marneffei infection. The present case suggested that clinical doctors and patients must pay more attention to regular treatment of the disseminated P. marneffei. It also highlighted the requirement for awareness of penicilliosis in non-acquired immune deficiency syndrome patients, who are not immunocompromised, who are living in or traveling to P. marneffei-endemic areas.
\end{abstract}

\section{Introduction}

Penicillium marneffei is the only Penicillium that is a dimorphic fungus in China and South-Eastern Asia. In 1956, $P$. marneffei was first time isolated from the hepatic lesions

Correspondence to: Professor Jun Liu, Department of Radiology, The Second Xiangya Hospital of Central South University, Middle Renmin Road 139, Changsha, Hunan 410000, P.R. China

E-mail: 2322349829@qq.com

Key words: disseminated Penicillium marneffei infection, therapy, disease recurrence of a bamboo-rat (Rhizomys sinensis). The disease can occur in healthy individuals; however, is more commonly detected in immunodeficient or immunnosuppressed patients. With the recently increasing incidence of HIV infection, reports of P. marneffei infection are increasing. It was one of the top three AIDS-defining opportunistic infections, alongside tuberculosis and cryptococcosis. Penicillium marneffei often violates multiple body organs, causes immune deficiency or immune suppression, and is becoming more common in recent years (1-3), and this trend will continue to deteriorate. Since the clinical manifestation is complicated and does not have very high specificity, this often leads to improper diagnosis, mistakes when deciding the correct treatment duration, and therefore, the infection is associated with a high mortality rate (4). Diagnosis of Penicillium fungal disease is predominantly isolated from clinical specimens of Penicillium marneffei, and after a thorough treatment it can be cured. Itraconazole and ketoconazole is the best choice for mild and moderate infection with P. marneffei, and amphotericin B may be effective in the treatment of severe infection with P. marneffei. To date, no cases of $P$. marneffei infection recurrence following treatment have been reported. The present study described the first case, to the best of our knowledge, of a non-acquired immune deficiency syndrome patient, who is not immunocompromised, with complications following $P$. marneffei infection without any strict treatment, causing disease recurrence. The present study described the details of the case with emphasis on the importance of standard treatment. Increased clinician and patient attention to the disease is essential to prevent any recurrence, even in non-immunocompromised patients.

\section{Case report}

A 36-year-old female, a native of Hunan province in central China, had a 2 month history of subcutaneous abscess and a 1 month history of intermittent fever, cough and anhelation. The patient was informed that she had been infected with P. marneffei 1 year previously. Since then, the masses had gradually increased both in size and numbers. Despite the use of therapy with various antibacterial antibiotics for tuberculosis, the granulomatous lesions in the left upper lobe of the lungs persisted, the condition failed to improve and gradually deteriorated. For further treatment, the patient was admitted to The Second Xiangya Hospital of Central South University (Changsha, China) on February 


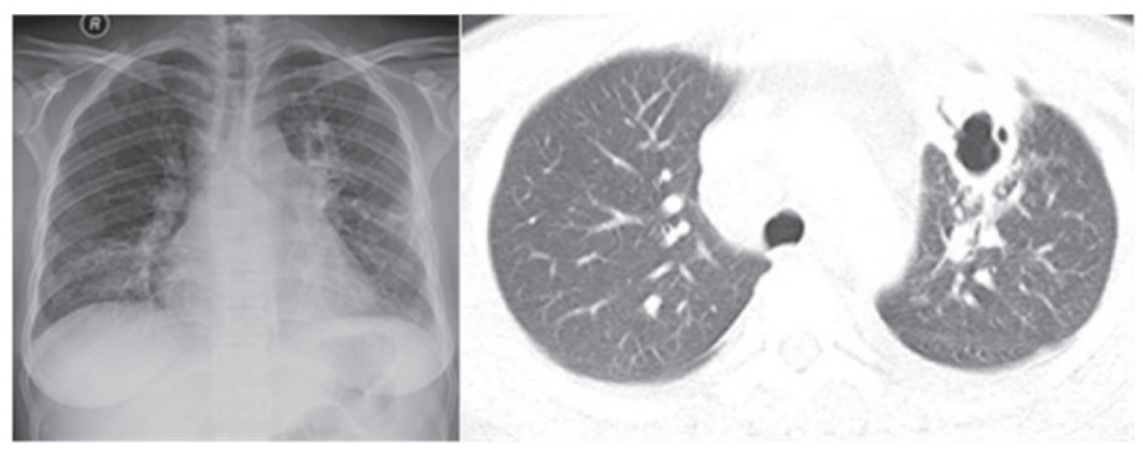

Figure 1. Chest radiographs and computed tomography scans revealed multiple patchy exudative shadows and a thick-walled hollow inner part of the visible lesions in the lung.

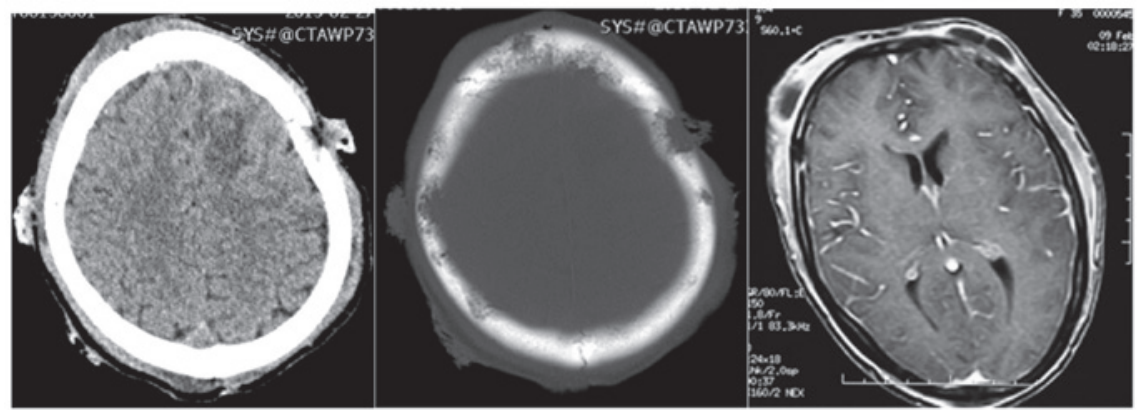

Figure 2. The computed tomography and enhanced magnetic resonance imaging revealed multiple sites of bone destruction an masses in the skull, with ring-enhanced abscesses in the scalp.

25th 2015. Physical examination revealed a body temperature of $39.1^{\circ} \mathrm{C}$, a pulse rate of 140 beats $/ \mathrm{min}$. Routine blood examination revealed the following: White blood cell count, $15.13 \times 10^{-9} / 1$ (normal level, $\left.3.5-9.5 \times 10^{9} / 1\right)$, red blood cell count, $3.39 \times 10^{12} / 1$ (normallevel,4.3-5.8 $\left.\times 10^{12} / 1\right)$ andhemoglobinlevel, $88 \mathrm{~g} / \mathrm{l}$ (normal level, 130-175 g/l). Chest radiographs and chest computed tomography revealed multiple patchy exudative shadows, thick-walled hollow inner part of the visible lesions, bilateral pleural and pericardial effusion in the lungs (Fig. 1).

The CT and enhanced MR show multiple bone destruction and masses in skull, with ring-enhanced abscesses in scalp (Fig. 2), and bilateral neck, bilateral upper arm, head and face have multiple visible purulent mass. A culture of blood and cyst fluid revealed P. marneffei infection (Fig. 3). The patient was administered 2 weeks of antifungal therapy, and the patient's condition was markedly improved. The exudative lesions significantly improved absorption and systemic multiple pustule subsided. Patients and families must consider the costs of treatment. It was recommended that the patient return to the local hospital for follow-up treatment following discharge from The Second Xiangya Hospital of Central South University on March 10th 2015. Following discharge, liposomal amphotericin B was used (initial dose, $25 \mathrm{mg} /$ day for 4 days, increasing to $150 \mathrm{mg}$ /day for 12 days, then the total applications of amphotericin B liposome was up to 2,000 mg). After three months of treatment, the abscesses of the head, face and other body parts disappeared and the enlarged lymph nodes disappeared. The sizes and number of lesions in the chest and abdomen were significantly reduced. The symptoms of the patient were significantly improved, so she decided
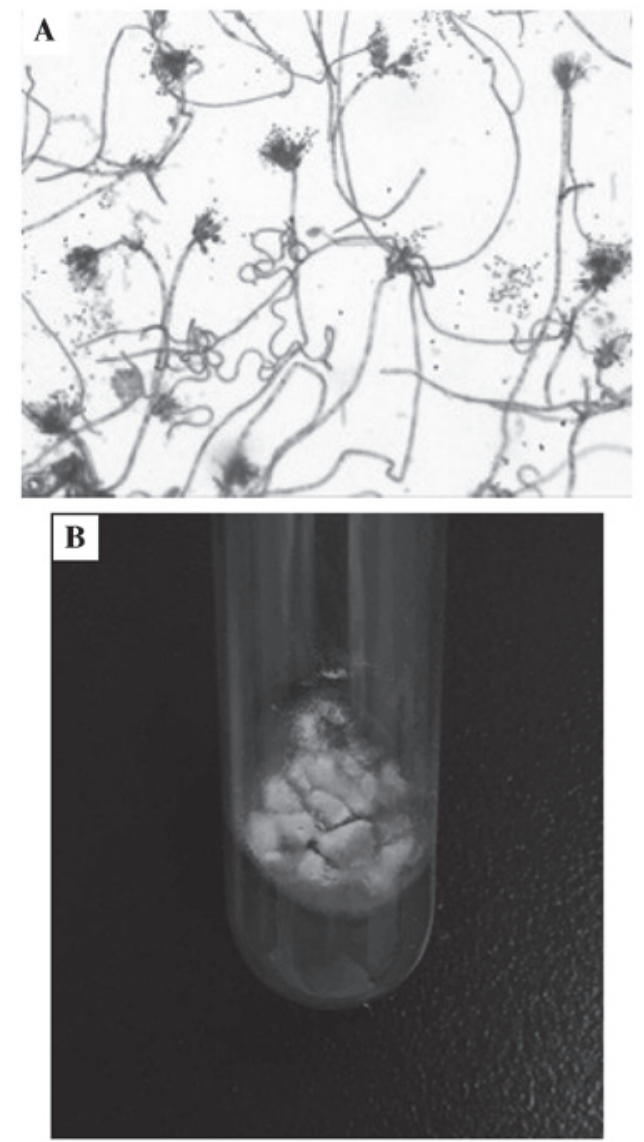

Figure 3. (A) Lacto-phenol cotton blue stain revealed Penicillium marneffei hyphae. (B) The Sabouraud medium at $25^{\circ} \mathrm{C}$ turns wine-red due to pigment created by Penicillium marneffei. 
to stop antifungal treatment without medical advice. At 10 months after stopping treatment, the abscesses in the head and neck, the left breast, and right leg reappeared. After the anti-tuberculosis treatment at the local hospital, the condition gradually deteriorated, and pleural and pericardial effusion was observed. The patient was re-admitted to The Second Xiangya Hospital of Central South University again. A culture of the systemic lesion abscess pus once again confirmed $P$. marneffei infection. After a period of antifungal therapy with amphotericin B (daily $0.6 \mathrm{mg} / \mathrm{kg}$ ) for 2 weeks of treatment, followed by oral itraconazole ( $400 \mathrm{mg} /$ day) maintenance therapy, disappearance of the abscesses were confirmed, body temperature returned to normal, and pleural and pericardial effusion disappeared. To date, recurrent disease has not been detected.

\section{Discussion}

The penicillium genus Marneffei is a dimorphic fungus. $P$. marneffei does not cause serious human health problems, but it can cause disease. This bacteria can be found widespread in nature (5). During the past few years, the incidence of penicilliosis infection has markedly increased, paralleling the incidence of human immunodeficiency virus infection. In China, the endemic of P. marneffei infection in South China, particularly in Guangdong, Guangxi, Yunnan and other regions (6). The source of infection is most likely decaying vegetative material or soil from natural habitat. In previous years, reports of immunocompromised patients infected with $P$. marneffe $i$ has gradually increased.

P. marneffei can cause three chemical reactions in tissues: Granulomatous reaction, purulent reaction and non-reactive necrosis. It can affect the body's major organs, the most common organs being the trachea, lung parenchyma, hematopoietic and lymphoid system, skin, mucous membranes, bone and soft tissue (7). As a result of a lack of specific clinical manifestations, misdiagnosis often occurs. P. marneffe $i$ diagnosis using tissue culture of bone marrow and lymph node specimens have a high positive rate and the highest reliability. Due to its histological culture lag, the treatment of patients is often delayed. The patients presented with fever, cough, multiple abscesses on the body surface and bone destruction. Antifungal therapy provides a period of rapid relief of clinical symptoms. However, this relief does not continue if the patient does not present for follow-up review and they voluntarily decide to stop taking the drug and return to factory jobs, as demonstrated in the present study. In February 2016, the present patient presented with abscesses of the head, neck and left breast. A culture of blood and cyst fluid revealed infection with $P$. marneffei once again. Treatment options were intravenous amphotericin B (daily $0.6 \mathrm{mg} / \mathrm{kg}$ ) for 2 weeks of treatment, followed by oral itraconazole (400 mg/day) maintenance therapy. To date, this patient with recurrent disease has not exhibited any recurrence (8).

Disseminated P. marneffei infection is relatively rare, with no reported cases ofrelapse until now. For immunocompromised patients, a lack of strict antifungal treatment and timely follow-up will result in repeated systemic multiple abscess and bone destruction. The disease development is rapid and often causes a high rate of mortality. If patients fail to maintain strict antifungal treatment and timely follow-up, it easily causes deterioration and even patient mortality. Therefore, Carlyle marneyfe penicillium consolidation treatment is necessary (9).

In conclusion, when a patient presents with systemic infection, $P$. marneffei infection must be considered, particularly due to the increase in incidence of $P$. marneffe $i$ in both immunocompromised and immunecompetent hosts. Increased attention by the clinician and patient is essential to manage the infection, and antifungal treatment is of great importance. To prevent recurrence, long-term effective antifungal therapy is notably important; therapy withdrawal requires strict and systemic evaluation. Treatment follow-up after the review is essential.

\section{References}

1. Saadiah S, Jeffrey AH and Mohamed AL: Penicillium marneffei infection in a non aids patient: First case report from Malaysia. Med J Malaysia 54: 264-266, 1999.

2. Yousukh A, Jutavijittum P, Pisetpongsa P, Chitapanarux T, Thongsawat S, Senba M and Toriyama K: Clinicopathologic study of hepatic Penicillium marneffei in Northern Thailand. Arch Pathol Lab Med 128: 191-194, 2004.

3. Cao C, Liang L, Wang W, Luo H, Huang S, Liu D, Xu J, Henk DA and Fisher MC: Common reservoirs for Penicillium marneffei infection in humans and rodents, China. Emerg Infect Dis 17: 209-214, 2011.

4. Skalski JH and Limper AH: Fungal, viral and parasitic pneumonias associated with human immunodeficiency virus. Semin Respir Crit Care Med 37: 257-266, 2016.

5. Li X, Yang Y, Zhang X, Zhou X, Lu S, Ma L, Lu C and Xi L: Isolation of Penicillium marneffei from soil and wild rodents in Guangdong, SE China. Mycopathologia 172: 447-451, 2011.

6. Hu Y, Zhang J, Li X, Yang Y, Zhang Y, Ma J and Xi L: Penicillium marneffei infection: An emerging disease in mainland China. Mycopathologia 175: 57-67, 2013.

7. Duong TA: Infection due to Penicillium marneffei, an emerging pathogen: Review of 155 reported cases. Clin Infect Dis 23: 125-130, 1996.

8. Patassi AA, Saka B, Landoh DE, Kotosso A, Mawu K, Halatoko WA, Wateba MI, Adjoh K, Tidjani O, Salmon D and Pitché P: First observation in a non-endemic country (Togo) of Penicillium marneffei infection in a human immunodeficiency virus-infected patient: A case report. BMC Res Notes 6: 506, 2013.

9. Kawila R, Chaiwarith R and Supparatpinyo K: Clinical and laboratory characteristics of penicilliosis marneffei among patients with and without HIV infection in Northern Thailand: A retrospective study. Bmc Infect Dis 13: 464, 2013. 